\title{
Intervención con probióticos para la prevención de enterocolitis necrotizante en prematuros extremos menores de 1500 gramos o de 32 semanas Probiotic intervention to prevent necrotizing enterocolitis in extremely preterm infants born before 32 weeks of gestation or with a birth weight of less than $1500 \mathrm{~g}$
}

\author{
Dr. César Gutiérrez Escárate ${ }^{a}$, Prof. Luis Bustos Medina ${ }^{b}$,Dra. Katherine Caniulao Ríosc, \\ Dra. Carolina Taito Antivil', Dra. Yessica Gallegos Casanova ${ }^{a}$ y \\ Dra. Camila Silva Beltrán
}

\section{RESUMEN}

Introducción. Existe evidencia del beneficio de los probióticos en prevenir enterocolitis necrotizante en prematuros extremos. Desde 2015, se usa probiótico preventivo en el Servicio de Neonatología, Hospital Hernán Henríquez Aravena, Temuco, Chile.

Objetivo. Evaluar el impacto de este probiótico en la incidencia, gravedad, necesidad de terapia quirúrgica y letalidad por enterocolitis necrotizante en prematuros extremos.

Pacientes y método. Estudio retrospectivo de cohortes. Datos analizados con Stata. Se aplicó la prueba exacta de Fisher para comparar porcentajes $\mathrm{y}$, para los promedios, la prueba $t$ para varianzas distintas. Los egresados entre2015 y 2017 recibieron Lactobacillus reuteri Protectis $(L R P)$, dosis única $\left(1 \times 10^{8}\right.$ unidades formadora de colonias) desde los primeros días de vida hasta cumplir las 36 semanas deedad gestacional corregida. Los controles egresados entre 2012 y 2014 no recibieron $L R P$.

Resultados. El 3,45\% de los casos tuvo algún grado deenterocolitis: grado (el64\%), II (el 18\%), III (el $18 \%$ ); requirió cirugía el $18 \%$ y no hubo letalidad. El 3,75 \% de los controles históricos presentaron enterocolitis: grado I (el $12 \%)$, II (el $35 \%$ ), III (el $53 \%$ ); el 64,7 \% requirió cirugía, y el $47 \%$ falleció. El grupo intervenido presentó grado II o III en un $36 \%$; en el grupo control, la sumatoria de estos estadios fue del $88 \%$.

Conclusión. LRP administrado en dosis única diaria al prematuro extremo no modificó la incidencia de enterocolitis, pero disminuyó su gravedad, la letalidad y necesidad de tratamiento quirúrgico.

Palabras clave: recién nacido extremadamente prematuro, enterocolitis necrotizante, probióticos, Lactobacillus reuteri Protectis.

http:/ / dx.doi.org/10.5546/ aap.2021.185

Texto completo en inglés:

http:/ / dx.doi.org/10.5546/ aap.2021.eng.185

Cómo citar: Gutiérrez Escárate C, Bustos Medina L, Caniulao Ríos K, Taito Antivil C, et al. Intervención con probióticos para la prevención de enterocolitis necrotizante en prematuros extremos menores de 1500 gramos o de 32 semanas. Arch Argent Pediatr 2021;119(3):185-191.

\section{INTRODUCCIÓN}

La enterocolitis necrotizante (necrotizing enterocolitis; NEC, por sus siglas en inglés) es la urgencia gastrointestinal más frecuente y más grave que se presenta en el neonato, especialmente, en el prematuro extremo (PE). Su patogenia es compleja y multifactorial. La incidencia es variable en distintos países y centros neonatales, con reportes del 7,5\%, ${ }^{1}$ variaciones y tendencia al aumento. ${ }^{2}$

En el PE en riesgo de NEC, se ha encontrado una colonización fecal anormal y retardo en el inicio de la colonización saprófita. ${ }^{3,4}$ Hay una relación inversa entre el riesgo de desarrollar NEC y el peso de nacimiento y la edad gestacional: menos del $10 \%$ de los niños que desarrollan NEC son de término. ${ }^{5}$

Los probióticos son organismos vivos que ayudan al buen estado de la flora intestinal; favorecen el desarrollo de la flora saprófita y obstaculizan el crecimiento de bacterias patógenas. Su uso preventivo de NEC en PE se menciona, frecuentemente, en la literatura médica, y la evidencia científica los cataloga como una herramienta importante en la reducción de la $N E C$ grave en los prematuros. ${ }^{6,7}$ La falta de consenso en su uso profiláctico rutinario se relaciona con el tipo de probiótico por usar, la dosis requerida, la modalidad de tratamiento y los efectos a largo plazo, lo que ha contribuido a sugerir la realización de mayores estudios. ${ }^{8,9}$ Los mecanismos de acción 
de los probióticos son conocidos: alta afinidad para adherirse a la mucosa intestinal, mejoran la función de la barrera epitelial, exclusión competitiva de los patógenos, inhibición de la adhesión del patógeno, producción de sustancias antimicrobianas y modulación del sistema inmune del huésped.

A fines del año 2014, se solicitó la compra de Lactobacillus reuteri Protectis (LRP), cuya cepa es DSM 17938 (BioGaia ${ }^{\circledR}$ ), al Comité de Farmacia del Hospital Dr. Hernán Henríquez Aravena (HHHA) de Temuco, Chile, para la utilización rutinaria en los PE. No existen publicaciones en Chile con el uso de $L R P$ en $N E C$, por lo tanto, podría ser de utilidad como información a otros centros neonatales. El objetivo del presente estudio fue evaluar el impacto que tuvo este probiótico en la incidencia de $N E C$, su grado, la necesidad de tratamiento quirúrgico y la letalidad en un grupo de PE, comparado con otro grupo que no lo recibió.

\section{PACIENTES Y MÉTODOS}

El diseño de la muestra fue una cohorte retrospectiva de $\mathrm{PE}$, que incluyó a recién nacidos menores de 32 semanas de gestación o peso menor de 1500 gramos, que iniciaron la alimentación durante la primera semana de vida, sin malformaciones, sin genopatías o patologías que impidieran su alimentación y que fueron atendidos en el Servicio de Neonatología del HHHA entre los años 2012 y 2017.

El HHHA de Temuco, en la Región de la Araucanía, Chile, es un hospital regional a donde se derivan los pacientes cuyo grado de complejidad no puede ser resuelto en otros centros hospitalarios de la región. A Neonatología ingresan pacientes nacidos en el mismo hospital o en otros de la región y, en algunos casos, provenientes de otras regiones, según la disponibilidad de hospitalización en la Red Nacional de Salud.

En nuestro Servicio, se estimula la alimentación con leche materna, comprobados sus efectos benéficos y preventivos en la NEC. Cuenta con elementos bioactivos, además de probióticos y prebióticos. Se administra calostro a la boca al PE desde el primer día de vida, suero glucosado endovenoso con aminoácidos, hasta la llegada de la nutrición parenteral total al día siguiente. El aumento de la alimentación enteral depende de la evolución clínica y la tolerancia, con el fin de lograr cuanto antes el retiro de la nutrición parenteral. Se utiliza leche materna de preferencia, fórmula de prematuro o ambas. La leche materna se fortifica al alcanzar un volumen

TABla 1. Estadios de enterocolitis necrotizante de Bell modificados por Walsh-Kliegman

\begin{tabular}{|c|c|c|c|c|}
\hline Estadio & Signos sistémicos & Signos abdominales & Signos radiológicos & Tratamiento \\
\hline $\begin{array}{l}\text { I A } \\
\text { Sospecha }\end{array}$ & $\begin{array}{l}\text { Inestabilidad térmica, } \\
\text { apnea, bradicardia. }\end{array}$ & $\begin{array}{l}\text { Aumento de residuo gástrico, } \\
\text { distensión abdominal leve, } \\
\text { sangre oculta en deposiciones. }\end{array}$ & Normal o íleo leve. & $\begin{array}{l}\text { Régimen cero. } \\
\text { Antibióticos por } 3 \text { días. }\end{array}$ \\
\hline $\begin{array}{l}\text { I B } \\
\text { Sospecha }\end{array}$ & Los mismos. & Sangre fresca por el recto. & Normal o íleo leve. & Igual a I A. \\
\hline $\begin{array}{l}\text { II A } \\
\text { NEC leve }\end{array}$ & $\begin{array}{l}\text { Los mismos que } \\
\text { en el estadio I. }\end{array}$ & $\begin{array}{l}\text { Distensión abdominal marcada, } \\
\text { ausencia de ruidos intestinales, } \\
\text { sangre abundante en las heces. }\end{array}$ & $\begin{array}{l}\text { Íleo, dilatación de las } \\
\text { asas intestinales, } \\
\text { neumatosis focal. }\end{array}$ & $\begin{array}{c}\text { Régimen cero, } \\
\text { antibióticos por 7-10 días. }\end{array}$ \\
\hline $\begin{array}{l}\text { II B } \\
\text { Moderada }\end{array}$ & $\begin{array}{l}\text { Acidosis leve. } \\
\text { Trombocitopenia. }\end{array}$ & $\begin{array}{l}\text { Edema de la pared abdominal. } \\
\text { Masa palpable y sensible. }\end{array}$ & $\begin{array}{l}\text { Neumatosis extensa, } \\
\text { gas en la vena porta. } \\
\text { Ascitis temprana. }\end{array}$ & $\begin{array}{l}\text { Régimen cero, } \\
\text { antibióticos por } 14 \text { días. }\end{array}$ \\
\hline $\begin{array}{l}\text { III A } \\
\text { Grave } \\
\text { acido } \\
\mathrm{h}\end{array}$ & $\begin{array}{l}\text { Apnea, } \\
\text { ventilación mecánica, } \\
\text { osis metabólica o respiratoria, } \\
\text { ipotensión, oliguria, CID. }\end{array}$ & $\begin{array}{l}\text { Aumento del edema } \\
\text { de la pared abdominal } \\
\text { con eritema e } \\
\text { induración. }\end{array}$ & $\begin{array}{l}\text { Ascitis prominente. } \\
\text { Asa intestinal fija, } \\
\text { sin aire libre. }\end{array}$ & $\begin{array}{c}\text { Régimen cero, } \\
\text { antibióticos por } 14 \text { días, } \\
\text { fluidos, inotrópicos, } \\
\text { asistencia respiratoria, } \\
\text { paracentesis. }\end{array}$ \\
\hline $\begin{array}{l}\text { III B } \\
\text { Grave }\end{array}$ & $\begin{array}{l}\text { III A más } \\
\text { deterioro rápido } \\
\text { y shock. }\end{array}$ & $\begin{array}{c}\text { Distensión abdominal grave, } \\
\text { ausencia de ruidos intestinales. } \\
\text { Edema de la pared, equimosis, } \\
\text { induración. }\end{array}$ & $\begin{array}{c}\text { Aire libre } \\
\text { subdiafragmático. } \\
\text { Neumoperitoneo. }\end{array}$ & $\begin{array}{l}\text { Igual a III A } \\
\text { más cirugía. }\end{array}$ \\
\hline
\end{tabular}

NEC: enterocolitis necrotizante; CID: coagulación intravascular diseminada. 
de alrededor de $50 \mathrm{ml} / \mathrm{kg} /$ día. Esta práctica alimentaria se realiza desde hace más de 10 años.

Se separaron los pacientes en dos grupos según el año del alta: grupo control, de 2012 a 2014, y grupo intervenido, de 2015 a 2017. Fueron excluidos los neonatos que no hubieran recibido el probiótico en forma adecuada o no cumplieran con los criterios mencionados al inicio.

El procedimiento de administración del probiótico lo realizó personal de enfermería directamente en la boca del PE, en dosis de 5 gotas de $L R P$ (que contenía $10^{8}$ unidades formadora de colonias -UFC-) una vez al día, con inicio entre el tercero y el séptimo día de vida y hasta cumplir las 36 semanas de edad gestacional corregida.

Se definió NEC la inflamación aguda del intestino delgado o grueso con diferentes niveles de intensidad según los criterios de Bell modificados por Walsh y Kliegman (Tabla 1). Probióticos: organismos vivos que ayudan al desarrollo de la flora intestinal normal. Prebióticos: fibras vegetales o de almidón que actúan aportando nutrientes. Microbiota intestinal: un conjunto de bacterias que viven en el intestino en relación armónica sin producir daño y aportan beneficios para el organismo.
Microbioma: la composición genética de toda la microbiota presente en los distintos sistemas del organismo.

La recolección de la información demográfica y clínica de los pacientes se hizo en forma retrospectiva a partir del año 2019, una vez recibida la aprobación del Comité de Ética, mediante la revisión de fichas clínicas y epicrisis de ambos grupos. La autorización del Comité de Ética Científica del Servicio de Salud Araucanía Sur fue recibida el 11 febrero de 2019.

Por tratarse de una terapia de rutina, no se solicitó consentimiento informado. El de ingreso estaba relacionado con la autorización para la hospitalización y las medidas terapéuticas que se requirieran. Tampoco fue posible solicitarlo al momento de reunir los datos porque ya no estaban los pacientes. Además, se conservó la privacidad de ellos en todo momento. Esta situación fue presentada al Comité de Ética del Hospital y al Comité de Ética Regional.

Los datos fueron analizados con Stata y se aplicó la prueba exacta de Fisher para comparar porcentajes $y$, para los promedios, la prueba $t$ para varianzas distintas. El nivel de significación utilizado fue del $5 \%$.

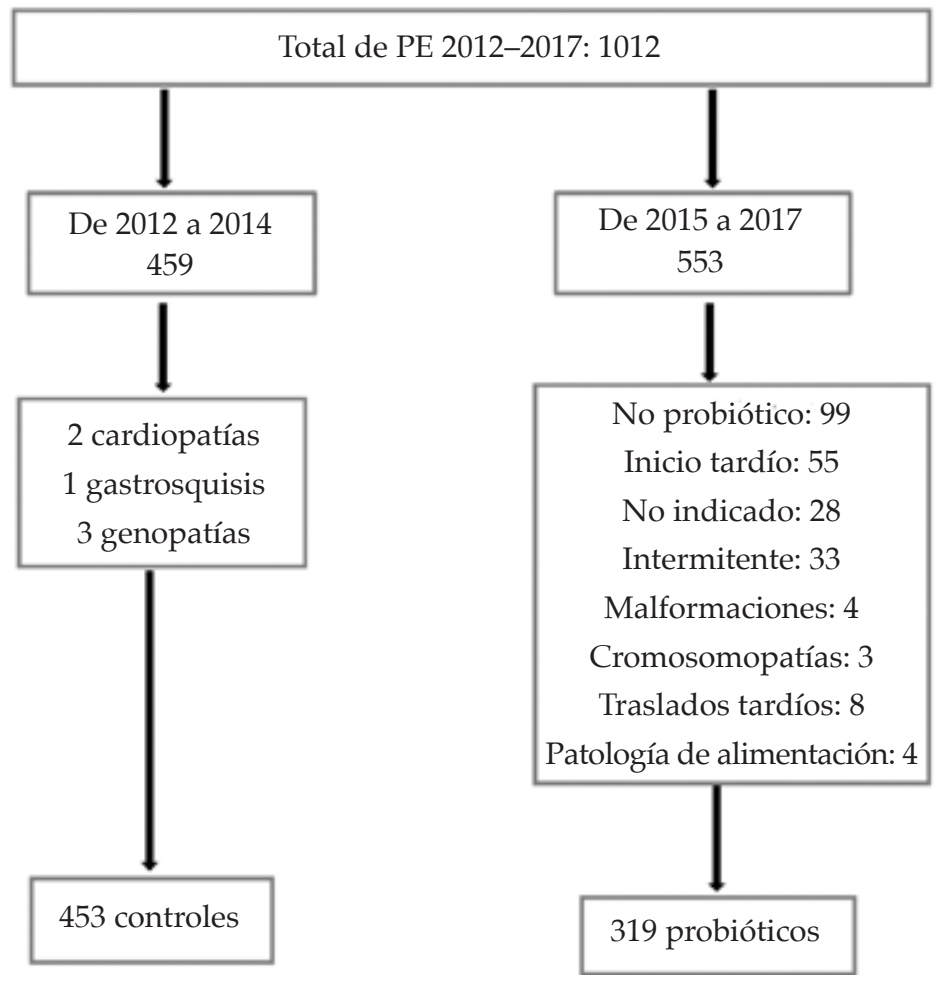

PE: prematuros extremos. 


\section{RESULTADOS}

La muestra de PE del estudio se encuentra en el flujograma (Figura 1). El peso promedio del grupo probiótico fue de 1318,28 gramos y, del control, 1213,93 gramos. La edad gestacional promedio del grupo probiótico fue 29,71 semanas y, en el control, 28,99 (desvío estándar -DE-: 2,49 ). Se encontraron asociaciones significativas con peso de nacimiento, edad gestacional, test de Apgar al minuto y a los 5 minutos, y con catéter venoso umbilical. En cambio, no hubo diferencias significativas en cuanto a sexo, rotura prematura de membranas, instalación de catéter arterial umbilical y transfusión de glóbulos rojos (GR) (Tabla 2).

De los 319 pacientes que recibieron probióticos, 11 (el 3,45\%) presentaron algún grado de NEC: el grado i B se observó en 7 de los 11 pacientes; el grado II ocurrió en 2 pacientes, y el grado III también fue encontrado en 2 pacientes. En cuanto al tipo de tratamiento, requirieron terapia quirúrgica 2 de los 11 neonatos, lo que equivalió a un $18 \%$ del total de pacientes de este grupo con NEC y no hubo mortalidad.

En el grupo control, 17 de los 453 presentaron NEC (el 3,75 \%) y la distribución fue la siguiente:

TABLA 2. Características de ambos grupos

\begin{tabular}{lccc}
\hline Grupo & $\begin{array}{c}\text { Probiótico: } \mathbf{n}=319 \\
\text { (año de 2015 a 2017) }\end{array}$ & $\begin{array}{c}\text { Control: } \mathbf{n}=\mathbf{4 5 3} \\
\text { (año de 2012 a 2014) }\end{array}$ & $p$ \\
\hline Peso promedio en g & 1318,28 (DE: 320,12) & 1213,93 (DE: 358,50) & $<0,0001^{*}$ \\
Edad gestacional promedio al nacer & 29,71 semanas (DE: 2,15) & 28,99 semanas (DE: 2,49) & $<0,0001^{*}$ \\
Test de Apgar 1 min. promedio & 6,97 puntos (DE: 2,21) & 6,52 puntos (DE: 2,19) & $0,0122^{*}$ \\
Test de Apgar 5 min. promedio & 8,64 puntos (DE: 1,28) & $8,21$ puntos (DE: 1,70$)$ & $0,0001^{*}$ \\
Catéter venoso & $273 / 319(85,58 \%)$ & $346 / 453(76,38 \%)$ & $0,002^{* *}$ \\
Sexo & F/M 159/160 $(49,84 / 50,16 \%)$ & F / M 205/248 $(45,25 / 54,75 \%)$ & $0,214^{* *}$ \\
Rotura prematura de membranas & $47 / 319(14,73 \%)$ & $92 / 453(20,31 \%)$ & $0,057^{* *}$ \\
Catéter arterial & $92 / 319(28,84 \%)$ & $130 / 453(28,70 \%)$ & $1^{* *}$ \\
Transfusión de glóbulos rojos & $184 / 319(57,68 \%)$ & $271 / 453(59,82 \%)$ & $0,553^{* *}$ \\
\hline
\end{tabular}

* Test para varianzas distintas; ** Prueba exacta de Fisher.

DE: desvío estándar.

FIGURA 2. Porcentajes de grados de enterocolitis: I (leve), II (moderada), III (grave)

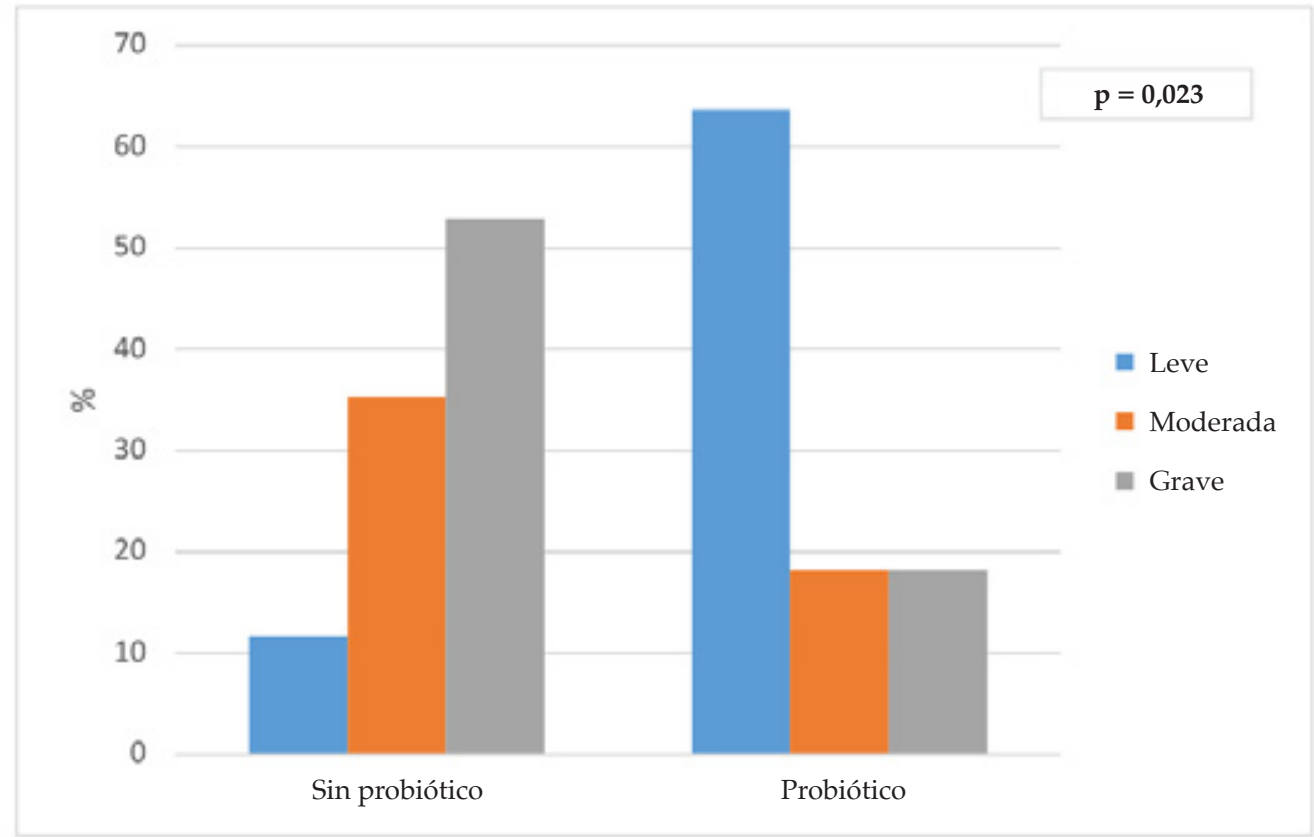

¿color o blanco y negro? 
el grado i B se presentó en 2 pacientes; el grado II, en 6 de ellos, y el grado III, en 9 de los 17 . Requirieron cirugía 11 de los 17, y la muerte por enterocolitis o a consecuencia de esta se presentó en 8 de ellos, lo que correspondió al $47 \%$ de los neonatos que presentaron NEC (Figura 2).

$\mathrm{Al}$ agrupar a los pacientes en los estadios más graves según los criterios de Bell modificados, se encontró que, en el grupo intervenido, la sumatoria de los grados II y III fue 4 de 11 pacientes (riesgo relativo-RR-: 0,41). En cambio, en el grupo control, la sumatoria de estos estadios se encontró en 15 de los 17 PE con NEC.

El esquema de tratamiento antibiótico de los PE que presentaron NEC fue similar en ambos grupos (ampicilina-amikacina en el grado i y se agregó metronidazol en el grado II y III). Además, se contó con protocolos de tratamiento con el fin de evitar su uso excesivo. La alimentación fue leche materna o mixta. Muy ocasionalmente, fórmula exclusiva.

\section{DISCUSIÓN}

En el presente estudio, se observó una baja incidencia de $N E C$ en ambos grupos, enterocolitis grave menos frecuente en el grupo probióticos, como también menor necesidad de cirugía y menos letalidad. Los pacientes con enfermedad grave fueron más frecuentes en el grupo control.

Nuestra terapia preventiva se apoya en su uso rutinario en algunos países europeos (además de Canadá y de Australia) para la prevención de NEC en PE. ${ }^{10-14}$ Dinamarca cuenta con guías clínicas para el uso de probióticos en el recién nacido prematuro. ${ }^{15}$ Una revisión sistemática de estudios observacionales ha confirmado los beneficios de los probióticos enterales en los neonatos prematuros. ${ }^{16}$

A partir de 2008, se estandarizó la administración de un probiótico (Lactobacillus casei rhamnosus, Lcr 35) a los PE del Hospital de la Universidad de Dijon, Francia. Un estudio retrospectivo de 3 años mostró la asociación entre la administración del probiótico y la disminución de NEC grave y mortalidad tardía, sin efectos adversos en la población tratada comparada con una cohorte histórica. ${ }^{17}$

La literatura médica científica continúa informando los resultados de sus estudios desde distintos aspectos estratégicos: la administración de cepas múltiples versus cepa única, ${ }^{18}$ la administración de probióticos como elemento único, la administración de probióticos más fructooligosacáridos, o pentoxifilina, o arginina y lactoferrina, como aditivos alimentarios. Estos estudios han sido realizados mediante un network metaanálisis. ${ }^{19}$ Últimamente, se ha planteado como la nueva frontera el uso de paraprobióticos, que correspondería a probióticos inactivados o trozos de ellos que producirían una respuesta beneficiosa en el organismo del recién nacido. ${ }^{20}$

Con respecto al Lactobacillus reuteri, probiótico aislado por primera vez en el año 1991 de la leche humana en una mujer peruana, se ha comprobado su colonización del tracto gastrointestinal y sobrevivencia a su paso por el conducto digestivo. Es productor de reuterina, una substancia antimicrobiana potente y de amplio espectro, que cuenta con estudios que demuestran efectos beneficiosos en el aparato digestivo del prematuro al disminuir infecciones gastrointestinales, sepsis y la estadía intrahospitalaria, ${ }^{21-23}$ y, en la prevención de $N E C$, se menciona un nivel de evidencia 2 en un estudio realizado por AthalyeJape en neonatos prematuros. ${ }^{24}$ Lactobacillus reuteri, además, produce cambios regulatorios en las células $\mathrm{T}$ del intestino y de los nódulos linfáticos mesentéricos, y mantiene la homeostasis intestinal, ya que restablece el déficit de células T reguladoras que están disminuidas en la NEC. Permite controlar la inflamación del intestino y mejora la tolerancia alimentaria, según estudios experimentales de NEC inducida en ratas. ${ }^{25,26}$

La incidencia de enterocolitis encontrada en ambos grupos fue inferior a la mencionada en la literatura en algunos trabajos de investigación. ${ }^{1,2}$ No hubo diferencias entre el grupo intervenido y el grupo control, lo que contrastó con algunos estudios en los que se encontró una disminución importante en la incidencia de NEC con el uso del probiótico. $^{27}$

La baja incidencia de NEC en nuestro Servicio comparada con lo publicado podría explicarse, en parte, por el aporte precoz de calostro a la boca a todo neonato ingresado al Servicio, especialmente, en estos PE. También por el libre acceso de las madres para visitar a sus hijos las 24 horas del día y los 7 días de la semana, y la extracción de leche al lado de la incubadora, lo que logra disminuir la tensión emocional de la madre y del niño, y facilita la alimentación con leche materna.

En cuanto a la gravedad de la enterocolitis, se encontró que la sumatoria de los grados II y III era menor en el grupo tratado que en el grupo control. La tendencia de este grupo fue a disminuir hacia los grados mayores (4 de 11 vs. 15 de 17 sumando II y III de Bell), al revés de lo 
ocurrido con los controles, que aumentaban hacia la gravedad. Fue concordante con lo descrito en la literatura para este mismo probiótico. ${ }^{27}$

La disminución en el requerimiento de cirugía tuvo relación directa con la menor gravedad presentada en el grupo tratado. La intervención quirúrgica fue el último nivel de la terapia y existió el riesgo de sufrir complicaciones asociadas a esta, ya fueran bridas, estenosis, suboclusiones, obstrucciones completas, fístulas o el temido "síndrome de intestino corto". El aspecto quirúrgico, aunque es poco mencionado en la literatura, en nuestro estudio, coincidió con lo publicado. ${ }^{27}$

$\mathrm{Al}$ analizar la letalidad de ambos grupos, se observaron diferencias entre ellos, ya que, para esta revisión, no se registró letalidad asociada a enterocolitis o como consecuencia de ella en el grupo intervenido, que contrastó con el $47 \%$ de letalidad por NEC que se presentó en el grupo control. Estos resultados difirieron de algunas publicaciones en que no hubo diferencias al usar el mismo probiótico. ${ }^{28,29}$

Fortalezas del presente estudio: ser el primero con este probiótico en Chile y haber obtenido resultados favorables para su utilización en neonatos.

Debilidades del estudio: ser retrospectivo de cohortes; no haber incluido datos sobre las patologías maternas o fetales causantes del parto prematuro; no haber analizado otras variables, como días de hospitalización, días de Unidad de Cuidados Intensivos (UCI), días de ventilación mecánica, de nutrición parenteral, complicaciones presentadas poscirugías.

\section{CONCLUSIONES}

Nuestros resultados apoyan que, con LRP administrado en una dosis única diaria a $P E$ desde el tercer día de vida y hasta las 36 semanas de edad gestacional corregida, si bien no se redujo la incidencia de $N E C$, se observaron menos casos de NEC grave. También se vio una menor necesidad de tratamiento quirúrgico y no hubo letalidad, en comparación con un grupo control de los 3 años anteriores.

\section{REFERENCIAS}

1. Robertson C, Savva GM, Clapuci R, Jones J, etal.Incidence of necrotising enterocolitis before and after introducing routine prophylactic Lactobacillus and Bifidobacterium probiotics. Arch Dis Child Fetal Neonatal Ed. 2020;105(4):380-6.

2. Horbar JH, Carpenter JH, Badger GJ, Kenny MJ, et al. Mortality and neonatal morbidity among infants 501 to 1500 grams from 2000 to 2009. Pediatrics. 2012;129(6):1019-26.

3. Gewolb IH, Schwalbe RS, Taciak VL, Harrison TS, et al.
Stool microflora in extremely low birthweight infants. Arch Dis Child Fetal Neonatal Ed. 1999;80(3):F167-73.

4. Goldmann DA, Leclair J, Macone A. Bacterial colonization of neonates admitted to an intensive care environment. J Pediatrics. 1978;93(2):288-93.

5. Nolan LS, Rimer JM, Good M. The role of human milk oligosaccharides and probiotics on the neonatal microbiome and risk of necrotizing enterocolitis: a narrative review. Nutrients. 2020;12(10):3052.

6. Alfaleh K, Anabrees J, Bassler D, Al-Kharfi T. Probiotics for prevention of necrotizing enterocolitis in preterm infants. Cochrane Database Syst Rev. 2011;(3):CD005496.

7. Morgan RL, Preidis GA, Kashyap PC, Weizman AV, et al. Probiotics reduce mortality and morbidity in preterm, low-birth-weight infants: a systematic review and network meta-analysis of randomized trials. Gastroenterology. 2020;159(2):467-80.

8. Braegger C, Chmielewska A, Decsi T, Kolacek S, et al Supplementation of infant formula with probiotics and/ or prebiotics: a systematic review and comment by the ESPGHAN. Committee on nutrition. J Pediatr Gastroenterol Nutr. 2011;52(2):238-50.

9. Shelby RD, Raab R, Besner GE, McElroy SJ. Hope on the horizon: promising novel therapies for necrotizing enterocolitis. Pediatr Res. 2020;88(Suppl 1):30-4.

10. Deshpande G, Rao S, Patole S. Probiotics in neonatal intensive care-Back to the future. Aust NZJObstet Gynaecol. 2015;55(3):210-7.

11. Härtel C, Pagel J, Rupp J, Bendiks M, et al. Prophylactic use of Lactobacillus acidophilus / Bifidobacterium infantis probiotics and outcome in very low birth weight infants. J Pediatr. 2014;165(2)285-9.

12. Janvier A, MaloJ, Barrington KJ. Cohort study of probiotics in a North American neonatal intensive careunit. J Pediatr. 2014;164(5):980-5.

13. Uberos J, Aguilera-Rodríguez E, Jerez-Calero A, MolinaOya M, et al. Probiotics to prevent necrotising enterocolitis and nosocomial infection in very low birth weight preterm infants. Br J Nutr. 2017;117(7):994-1000.

14. Patole SK, Rao SC, Keil AD, Nathan EA, et al. Benefits of Bifidobacterium breve M-16V supplementation in preterm neonates-A retrospective cohort study. PLoS One. 2016;11(3):e0150775.

15. Danish National Guideline. Probiotics for preterm GA 30 weeks [in Danish]. [Consulta: noviembre de 2011]. Disponible en: http:/ / www.rh-vejledninger.dk/ C1256FF60 028FE3A/0/FADF649DDFCAFF2FC12576A B0030868D? OpenDocument\&Highlight_2.

16. Olsen R, Greisen G, Schroder M, Brok J. Prophylactic probiotic for preterm infants: a systematic review and meta-analysis of observational studies. Neonatology. 2016;109(2):105-12.

17. Bonsante F, Iacobelli S, Gouyon JB. Routine probiotic use in very preterm infants: Retrospective comparition of two cohorts. Am J Perinatol. 2013;30(1):41-6.

18. Chang HY, Chen JH, Chang JH, Lin HC, et al. Multiple strains probiotics appear to be the most effective probiotics in the prevention of necrotizing enterocolitis and mortality: An update meta-analysis. PLoS One. 2017;12(2):e0171579.

19. Yu W, Sui W, Mu L, Yi W, et al. Preventing necrotizing enterocolitis by food additives in neonates. A network meta-analysis revealing the efficacy and safety. Medicine (Baltimore). 2017;96(21):e6652.

20. Deshpande G, Athalye-Jape G, Patole S. Para-probiotics for preterm neonates, the next frontier. Nutrients. 2018;10(7):871.

21. Rojas MA, Lozano JM, Rojas MX, Rodriguez VA, et al. Prophylactic probiotics to prevent death and nosocomial 
infection in preterm infants. Pediatrics. 2012;130(5):e1113-20.

22. Romeo MG, Romeo DM, Trovato L, Oliveri S, et al. Role of probiotics in the prevention of the enteric colonization by Candida in preterm newborns: incidence of late-onset sepsis and neurological outcome. J Perinatol.2011;31(1):63-9.

23. Indrio F, Riezzo G, Raimondi F, Bisceglia M, et al. The effects of probiotics on feeding tolerance, bowel habits, and gastrointestinal motility in preterm newborns. J Pediatr. 2008;152:801-6.

24. Athalye-Jape G, Rao S, Patole S. Lactobacillus Reuteri DSM 17938 as a probiotic for Preterm Neonates: A StrainSpecific Systematic Review. JPEN J Parenter Enteral Nutr. 2016;40(6):783-94.

25. Liu Y, Fatheree N, Mangalat N, Rhoads JM. Lactobacillus reuteristrains reduceincidence and severity of experimental necrotizing enterocolitis via modulation of TLR4 and NF$\mathrm{kB}$ signaling in the intestine. Am J Physiol Gastrointest Liver Physiol. 2012;302(6):G608-17.
26. He B, Hoang TK, Wang T, Ferris M, et al. Resetting microbiota by Lactobacillus reuteri inhibits T reg deficiencyinduced autoimmunity via adenosine $\mathrm{A}_{2 \mathrm{~A}}$ receptors. J Exp Med. 2017;214(1):107-23.

27. Hunter C, Dimaguila MA, Gal P, Wimmer J Jr, et al. Effect of routine probiotic, Lactobacillus reuteri DSM 17938, use on rates of necrotizing enterocolitis in neonates with birthweight $<1000$ grams: a sequential analysis. BMC Pediatr. 2012;12:142.

28. Hernández-Henríquez N, Rosas-Sumano AB, MonzoyVentre MA, Galicia-Flores L. Lactobacillus reuteri DSM 17938 en la prevención de enterocolitis necrosante en recién nacidos prematuros. Estudio piloto de eficacia y seguridad. Rev Mex Pediatr. 2015;81(2):37-43.

29. OncelMY, Sari FN, ArayiciS, Guzoglu N, etal. Lactobacillus Reuteri for the prevention of necrotising enterocolitis in very low birthweight infants: a randomised controlled trial. Arch Dis Child Fetal Neonatal Ed. 2014;99(2):F110-5. 